\title{
Stochastic volatility with heterogeneous time scales
}

\author{
Danilo Delpini ${ }^{1,2}$ and Giacomo Bormetti ${ }^{3,4}$ \\ ${ }^{1}$ Department of Economics and Business (DiSEA), University of Sassari, via \\ Muroni 25, 07100 Sassari, Italy \\ ${ }^{2}$ IMT Institue for Advanced Studies, P.za San Ponziano 6, 55100 Lucca, Italy \\ 3 Scuola Normale Superiore, Piazza dei Cavalieri 7, 56126 Pisa, Italy \\ ${ }^{4}$ INFN - Sezione di Pavia, via Bassi 6, 27100 Pavia, Italy \\ E-mail: ddelpini@uniss.it
}

\begin{abstract}
Agents' heterogeneity is recognized as a driver mechanism for the persistence of financial volatility. We focus on the multiplicity of investment strategies' horizons, we embed this concept in a continuous time stochastic volatility framework and prove that a parsimonious, two-scale version effectively captures the long memory as measured from the real data. Since estimating parameters in a stochastic volatility model is challenging, we introduce a robust methodology based on the Generalized Method of Moments supported by a heuristic selection of the orthogonal conditions. In addition to the volatility clustering, the estimated model also captures other relevant stylized facts, emerging as a minimal but realistic and complete framework for modelling financial time series.

PACS numbers: 02.50.-r, 05.10.Gg, 89.65.Gh
\end{abstract}

Submitted to: Journal of Statistical Mechanics: Theory and Experiment

\section{Introduction}

In 1963 [1, 2] Benoît Mandelbrot refers to the volatility clustering as "large changes tend to be followed by large changes, of either sign, and small changes tend to be followed by small changes". Since then this effect has remained one of the most intriguing properties exhibited by financial time series. In the early Nineties the long memory property of absolute stock market returns was independently investigated by [3] and [4. In the former work, after amending absolute price changes from the heteroscedasticity due to seasonal effects, the authors find a persistent positive autocorrelation declining hyperbolically with the time lag. In the latter, analysing the daily closing prices of Standard\&Poor 500 index for the time span January 31928 - August 30 1991, Ding and collaborators study the power correlation of absolute returns $\left|r_{t}\right|^{d}$ for positive $d$, finding a strong persistence especially for $d$ close to one.

The slow decay of the volatility can be ascribed to two rather different mechanisms. Agent Based Models provide a first explanatory framework, where macroscopic evidences are explained in terms of microscopic interactions among market participants. As clarified in the seminal papers [5, 6] the alternation of the economic agents between chartist and fundamentalist regime can be identified as 
the source of the observed volatility clustering, an empirical signature of persistence. The same mechanism leading to the previous regime switching is further investigated in [7, 8], where the minimal assumptions required for an agent based model to capture the empirical stylized facts are identified. In a different approach 9 persistence is induced by the coexistence of agents differing in their perceptions of the market, risk profiles, institutional constraints, degree of information, prior beliefs, and other characteristics such as geographical locations. In [10] the role of heterogeneous time horizons for the investment strategies is specifically addressed. In [11] the daily, weekly and monthly time scales are isolated as the relevant ones, while the first direct evidence of these three scales as well as an attempt to capture them with an ARCH model is provided in [12. As a major achievement of the latter work we see that a small subset of time scales succeeds in capturing the long run behaviour of the squared return correlation. Interestingly, those horizons reflect typical time scales of the human activity, which noticeably follow a pseudo-geometric progression [13. Generalizing the concept of a finite mixture of time scales to a continuum of agents, an attractive intuition is that the integrated effect of exponential heterogeneous strategies may lead to persistence. On a formal basis, this amounts to expressing the correlation function as

$$
C(\tau)=\int_{0}^{1 / \tau_{\min }} \exp \left(-\tau / \tau_{\text {agent }}\right) p\left(1 / \tau_{\text {agent }}\right) \mathrm{d}\left(1 / \tau_{\text {agent }}\right),
$$

which at the leading order for $\tau \rightarrow+\infty$ is determined by the behaviour of the density $p\left(1 / \tau_{\text {agent }}\right)$ around the origin. Indeed, by virtue of Watson's Lemma, we obtain $C(\tau) \sim 1 / \tau^{1+\alpha}$ provided that $p\left(1 / \tau_{\text {agent }}\right) \sim \tau_{\text {agent }}^{-\alpha}$ with $\alpha>-1$.

As far as the distributional properties of the volatility proxies are concerned, in [14 the inverse gamma distribution is identified as an effective approximation for both the low and high volatility regimes. The simplest model reproducing this distribution as a result of a volatility feedback effect corresponds to an ARCH-like equation which, in the continuous time limit, reads as a Langevin equation

$$
\frac{\mathrm{d} \sigma}{\mathrm{d} t}=-\kappa\left(\sigma-\sigma_{\infty}\right)+\eta \sigma \zeta(t),
$$

with $\kappa, \sigma_{\infty}, \eta$ positive constants. For this specific case, the stationary distribution of the volatility has the form of an inverse gamma

$$
\frac{\lambda^{\nu}}{\Gamma(\nu)} \frac{\mathrm{e}^{-\lambda / \sigma}}{\sigma^{1+\nu}}
$$

with $\nu=1+2 \kappa / \eta^{2}$ and $\lambda=2 \kappa \sigma_{\infty} / \eta^{2}$. In the following Section we propose an approach inspired by this evidence about the volatility, as well as by the idea of a mixture of heterogeneous investment horizons, in a spirit similar to the Heston multifactor model [15].

The remainder of the paper is organized as follows: in Section 3 we derive analytical expressions for the leverage and volatility autocorrelation, while in Section 4 we detail a calibration procedure which is inspired by the Generalized Method of Moments. We conclude in Section 5 . The analytical derivations are postponed in the three Appendices at the end of the paper. 


\section{The model}

A quite general expression for the asset price at time $t$, reminiscent of the Geometric Brownian motion paradigm, is given by

$$
S_{t}=S_{0} \exp \left(\mu t+X_{t}\right)
$$

where $X_{t}$ is the stochastic centred log-return and $\mu$ a constant drift coefficient. In 16 we assume that the time evolution of $X_{t}$ can be modelled in terms of the stochastic differential equation (SDE)

$$
\mathrm{d} X_{t}=\sigma_{t} \mathrm{~d} W_{t}^{X},
$$

where $\sigma_{t}$ is the instantaneous volatility of the price and $\mathrm{d} W_{t}^{X}$ the increment of a standard Wiener process. Since $X_{0}$ is equal to zero, we also have $\mathbb{E}\left[X_{t}\right]=0$ and $\mathbb{E}\left[\ln S_{t}-\ln S_{0}\right]=\mu t$ for any $t$. A common choice accounting for the stochastic behaviour of the volatility, as measured by suitable proxies, is $\sigma_{t}=\sigma\left(Y_{t}\right)$ as a function of an unobserved driving process $Y_{t}$. General financial considerations regarding the mean-reverting behaviour of the volatility process lead to a second SDE of the form

$$
\mathrm{d} Y_{t}=-\kappa_{Y}\left(Y_{t}-y_{\infty}\right) \mathrm{d} t+\sqrt{\Sigma\left(Y_{t}\right)} \mathrm{d} W_{t}^{Y},
$$

with $\kappa_{Y}=1 / \tau_{Y}>0$, and $y_{\infty}>0$. In [16] $\Sigma\left(Y_{t}\right)$ is equal to $\sigma_{Y}^{2} Y_{t}^{2}$ with $\sigma_{Y}>0$, from which it follows that $\sigma_{t}$ is proportional to $Y_{t}$. This choice leads to an inverse gamma stationary distribution with shape and scale parameters $\nu=1+2 \kappa_{Y} / \sigma_{Y}^{2}$ and $\lambda=2 \kappa_{Y} y_{\infty} / \sigma_{Y}^{2}$, respectively; in light of the considerations presented in the Introduction, this is dictated by the will of recovering the most effective statistical description of the volatility distribution. Different choices for $\Sigma$ have been suggested in the literature and among the most popular ones it is worth mentioning the Heston 17 and Stein-Stein 18 models. For a complete overview of continuous time models as well as widely employed discrete time approaches like ARCH, GARCH and their generalizations, we suggest the handbook about financial time series [19.

Following the spirit of the Introduction, in this paper we extend the model given by (1) and (2) allowing the instantaneous volatility $\sigma_{t}$ to depend on multiple stochastic unobserved factors. Before stating explicitly our model's equations, it is worth noting that such a generalization is inspired by the multi-factor stochastic volatility model introduced by Bates in [15] as a possible description of the S\&P 500 futures price, and later revisited in 20 as a model for the dynamics of the volatility smirk in the

option pricing context. In the generalized form used in [21, the multi-factor model with jumps reads

$$
\begin{aligned}
\mathrm{d} X_{t} & =\sum_{i=1}^{N} \sqrt{Y_{t}^{i}} \mathrm{~d} W_{t}^{i}+\mathrm{d} J_{t}^{X} \\
\mathrm{~d} Y_{t}^{i} & =-\kappa_{i}\left(Y_{t}^{i}-y_{\infty}^{i}\right) \mathrm{d} t+\eta_{i} \sqrt{Y_{t}^{i}} \mathrm{~d} W_{t}^{i+N}+\mathrm{d} J_{t}^{i}, \quad i=1, \ldots, N
\end{aligned}
$$

where $W_{t}^{1}, \ldots, W_{t}^{2 N}$ is a multivariate possibly correlated Brownian motion and $\left\{J^{X}, J^{1}, \ldots, J^{N}\right\}$ is a multivariate possibly correlated Poisson process with constant intensities. In principle, each factor may be linked to the sensitivity of the economic agents to different investment horizons, and in light of this heterogeneity the modelling could reflect $N$ volatility components. Our starting point is a special case of the dynamics (3): we take $N=2$, discard the jump contribution and assume perfect 
correlation between the Wiener processes of the two factors, $\operatorname{corr}\left(\mathrm{W}_{\mathrm{t}}^{2}, \mathrm{~W}_{\mathrm{t}}^{2}\right)=1$. However, at variance with equation (3) and as a major contribution of our paper, we consider inverse gamma driving factors, each one being described by the same mean-reverting dynamics provided in (2) with $\Sigma$ proportional to the squared process. Ultimately the model we are going to analyse reduces to

$$
\begin{aligned}
& \mathrm{d} X_{t}=Y_{t} \mathrm{~d} W_{t}^{X}+Z_{t} \mathrm{~d} W_{t}^{X} \\
& \mathrm{~d} Y_{t}=-\kappa_{Y}\left(Y_{t}-y_{\infty}\right) \mathrm{d} t+\sigma_{Y} Y_{t} \mathrm{~d} W_{t}^{Y} \\
& \mathrm{~d} Z_{t}=-\kappa_{Z}\left(Z_{t}-z_{\infty}\right) \mathrm{d} t+\sigma_{Z} Z_{t} \mathrm{~d} W_{t}^{Z},
\end{aligned}
$$

where we impose the initial time conditions $X_{t=0}=X_{0}=0, Y_{t=t_{0}}=y_{0}>0$ and $Z_{t=t_{0}}=z_{0}>0$, with $\kappa_{Y}=1 / \tau_{Y}>0$, and $\kappa_{Z}=1 / \tau_{Z}>0$. We also indicate $\nu_{Y}=1+2 \kappa_{Y} / \sigma_{Y}^{2}$ and $\nu_{Z}=1+2 \kappa_{Z} / \sigma_{Z}^{2}$ the tail exponents of the inverse gamma stationary distributions of $Y_{t}$ and $Z_{t}$. The correlation structure among the three Brownian motions is described by the following matrix

$$
\left(\begin{array}{ccc}
1 & \rho_{X Y} & \rho_{X Z} \\
\rho_{X Y} & 1 & \rho_{Y Z} \\
\rho_{X Z} & \rho_{Y Z} & 1
\end{array}\right) .
$$

It has to be noted that we assume different starting times for the volatility factors and the return process, according to what is done in [16]. Indeed, as the processes $Y_{t}$ and $Z_{t}$ are unobserved factors and we are mainly concerned with their dynamics at the stationary state, we assume they start at $t_{0}<0$ in the past and we recover the stationary limit by letting $t_{0} \rightarrow-\infty$. On the other hand, $X_{t}$ represents the observed (detrended) logarithmic increment of the price for a fixed time lag and, therefore, it seems natural to take the spot time $t=0$ as a starting time for this lag.

Some considerations are due regarding our choice of the factors specification. In [16] the single factor $Y_{t}$ corresponds (up to a constant) to the instantaneous volatility itself. As such, $Y_{t}$ has a clear interpretation and its dynamics is chosen specifically with the intent to accommodate the distributional properties of the volatility observed in the reality. Here, in the spirit of the factor model (3), the evolution of log-returns is given in terms of two additive factors; it follows that $Y_{t}$ and $Z_{t}$ can not be interpreted, separately, as the return volatility (or possibly the variance as in $\mathrm{ARCH} / \mathrm{GARCH}$ models), but as the underlying unobserved factors. The choice of full correlation between $W_{t}^{1}$ and $W_{t}^{2}$ allows us to introduce formally $\sigma_{t}=Y_{t}+Z_{t}$ and to motivate the inverse gamma dynamics which drives the factors. Implicitly, this means that we give up recovering exactly an asymptotic inverse gamma law for $\sigma_{t}$ and we give priority to capturing the observed, long range memory of the squared return correlation. Nevertheless, we expect the tail asymptotic to be preserved under suitable assumptions (see discussion at the end of this Section). Finally, we observe that generalization with more that two factors is straightforward, but cumbersome, and would greatly simplify if we assume $\operatorname{corr}\left(W_{t}^{1}, W_{t}^{2}\right)=0$ in (3). However, the specific purpose of this work is to show how our minimal choice is indeed able to capture the very consequences of heterogeneity.

From [16] we know that a negative $\rho_{X Y}$ suffices to accommodate the observed short range scaling of the return-volatility correlation; in Appendix B and in the numerical section we set $\rho_{X Z}$ equal to zero to prevent $Z_{t}$ from impacting the leverage. Nonetheless, in what follows we derive the relation between the factors behaviour and the moments of $X_{t}$ under the general case of non trivial correlations between the Brownian motions. As we show in Appendix A, the structure of the model (4) 
allows to compute the moments of the probability density function (PDF) of $X_{t}$ at all times $t$ recursively. After cumbersome calculations, and by exploiting Itô's Lemma to compute the cross correlations between the two volatility factors, it can be verified that the moments of $X$ can be expressed always as a superposition of exponential functions of $\left(t-t_{0}\right)$

$$
\mathbb{E}\left[X_{t}^{n}\right]=\sum_{i, j=0 ; i+j \leq n}^{n} H_{i, j}^{(n)}\left(t ; y_{0}, z_{0}\right) \exp \left(F_{i, j}\left(t-t_{0}\right)\right),
$$

where the constants read $F_{m, n}=F_{m}^{Y}+F_{n}^{Z}+m n \rho_{Y Z} \sqrt{\sigma_{Y}^{2} \sigma_{Z}^{2}}$, with $F_{m}^{Y}=-\kappa_{Y} m+$ $m(m-1) \sigma_{Y}^{2} / 2$, and $F_{n}^{Z}=-\kappa_{Z} n+n(n-1) \sigma_{Z}^{2} / 2$. The coefficients $H_{i, j}^{(n)}$ depend on the time lag $t$; more precisely, due to the linearity of the ODEs A.2), they correspond to a combination of exponential terms weighted by polynomials in $t$.

In the following, we report the explicit expressions of the coefficients $H_{i, j}^{(n)}\left(t ; y_{0}, z_{0}\right)$ for the case $n=2$ (the constants $k_{i, j}^{(m, n)}$, which depend on the initial conditions $y_{0}, z_{0}$, are defined recursively in Appendix A

$$
\begin{aligned}
& H_{0,0}^{(2)}=\left[k_{0,0}^{(2,0)}+2 k_{0,0}^{(1,1)}+k_{0,0}^{(0,2)}\right] t, \\
& H_{1,0}^{(2)}=\left[k_{1,0}^{(2,0)}+2 k_{1,0}^{(1,1)}\right] \frac{1-\exp \left(-F_{1,0} t\right)}{F_{1,0}}, \\
& H_{0,1}^{(2)}=\left[k_{0,1}^{(2,0)}+2 k_{0,1}^{(1,1)}\right] \frac{1-\exp \left(-F_{0,1} t\right)}{F_{0,1}}, \\
& H_{2,0}^{(2)}=k_{2,0}^{(2,0)} \frac{1-\exp \left(-F_{2,0} t\right)}{F_{2,0}} \\
& H_{1,1}^{(2)}=2 k_{1,1}^{(1,1)} \frac{1-\exp \left(-F_{1,1} t\right)}{F_{1,1}} \\
& H_{0,2}^{(2)}=k_{0,2}^{(0,2)} \frac{1-\exp \left(-F_{0,2} t\right)}{F_{0,2}}
\end{aligned}
$$

Since $t$ is finite, the coefficients $H_{i, j}^{(n)}$ are finite quantities themselves, and all the relevant information about the behaviour of $\mathbb{E}\left[X_{t}^{n}\right]$ in the stationary limit of $Y$ and $Z$ is retained by the $t_{0}$-exponentials in Equation (5). Given that $F_{0,0}=0$, if all the $F_{i, j}$ for $i, j=0, \ldots, n$ with $i+j \leq n$ are negative, $\mathbb{E}\left[X_{t}^{n}\right]$ is finite in the stationary limit $t_{0} \rightarrow-\infty$, otherwise it diverges indicating the emergence of fat tails in the PDF $p_{t}(x)$ of $X_{t}$. In the latter case the tail behaviour would be compatible with an hyperbolic scaling with a tail exponent smaller than the order of the lowest diverging moment.

In [16] the hyperbolic scaling of $p_{t}(x)$ is induced by the power-law tail of the asymptotic (inverse gamma) distribution of the volatility, and a simple relation exists between the tail exponent of the latter and the order of the first diverging moment of $p_{t}(x)$. In the present case, the asymptotic distribution of $\sigma_{t}$ is that of the sum of the two factors $Y$ and $Z$, both inverse gamma distributed with tail indices $\nu_{Y}$ and $\nu_{Z}$ respectively. In the limit of $Y$ independent of $Z$ the distribution of the sum behaves as a power-law with tail index $\nu_{\sigma}=\min \left\{\nu_{Y}, \nu_{Z}\right\}=\nu$, see e.g. [22, 23]. Therefore, the same mechanism discussed in [16, which triggers the divergence of the return moments, applies here asymptotically in the absolute value $|X|$, and the PDF $p_{t}(x)$ manifests a decay compatible with a power-law scaling with tail index determined by the value of $\nu_{\sigma}$.

$\ddagger$ In fact, we make this assumption when estimating the model from the empirical data in Section 4 
The theoretical PDF is also compliant with the more basic properties of the returns. In particular, the stationary limit of $(5)$, and the expression of $H_{0,0}^{(2)}$, show that the variance is linear in the return time lag $t$. Furthermore, the explicit expressions of the functions $H_{0,0}^{(3)}$ and $H_{0,0}^{(4)}$, not reported here for the sake of parsimony, would also reveal that the skewness and kurtosis vanish in the limit of large $t$, according to the observed Gaussian-like shape of the distribution for large time horizons.

\section{Non linear dependence}

In this Section we discuss the main properties of the return correlation structure predicted by model (4), focusing on the return-volatility and the squared-return correlation functions.

Model (4) inherits from the class of stochastic volatility models the important property of absence of serial correlation, which is verified empirically with good approximation. Despite this, financial returns can not just follow a random walk process, since this would imply independent and identically distributed price increments. Any non linear function of the returns would then exhibit zero autocorrelation, a property that simply does not hold in practice. Empirical evidences of this violation are the leverage effect and the volatility clustering. The former refers to the negative correlation between past returns and the future instantaneous volatility, measuring the tendency of the market volatility to increase after a price downfall [24, 22, 25]; volatility clustering is usually expressed in terms of the persistent correlation between squared returns or logarithm of absolute returns, implying that large variations are more likely to be followed by large than small ones $[3,26,27,28,29$. For a survey of contributions on the same topics from the econometric community, we refer the interested readers to the reference list in [13, 30. Our model deals explicitly with these non linear correlation functions; their expressions, whose derivation we postpone to Appendix A-Appendix C, provide a valuable analytical characterization of model (4). This information can be exploited for the calibration of the model from empirical data, but it is also crucial to grasp the relevant information about the process time scaling, as we discuss in the rest of this Section.

\subsection{Leverage effect}

The leverage, a measure of the correlation between returns and volatility, is usually defined as $\mathcal{L}(\tau ; t)=\mathbb{E}\left[\mathrm{d} X_{t} \mathrm{~d} X_{t+\tau}^{2}\right] / \mathbb{E}\left[\mathrm{d} X_{t}^{2}\right]^{2}$. Empirically and for arbitrary $t$, $\mathcal{L}(\tau ; t)$ has been found to be negative and exponentially decaying for positive $\tau$ and approximately zero otherwise: a correlation exists between past returns and the volatility in the future and not vice versa.

For our case, a finite time, exact expression is derived in Appendix B; Equation B.2 reveals that the leverage function is characterized by the superposition of three exponential functions, with different characteristic times $\tau_{\mathcal{L}}, \tau_{\sim \mathcal{L}}, \tau_{<}$, which are ordered according to the following hierarchy:

$$
\begin{aligned}
& \tau_{\mathcal{L}}=\frac{2}{2-\tau_{Y} \sigma_{Y}^{2}} \tau_{Y}=\frac{\nu_{Y}-1}{\nu_{Y}-2} \tau_{Y} \\
& \tau_{\sim \mathcal{L}}=\left(\frac{1}{\tau_{Y}}-\frac{\sigma_{Y}^{2}}{2}+\frac{1}{\tau_{Z}}\right)^{-1}=\frac{\tau_{Z}}{\tau_{Z}+\tau_{\mathcal{L}}} \tau_{\mathcal{L}}<\tau_{\mathcal{L}}
\end{aligned}
$$




$$
\tau_{<}=\left[2\left(\frac{1}{\tau_{Y}}-\frac{\sigma_{Y}^{2}}{2}\right)-\frac{\sigma_{Y}^{2}}{2}\right]^{-1}=\frac{2}{4-\tau_{\mathcal{L}} \sigma_{Y}^{2}} \tau_{\mathcal{L}}=\frac{\nu_{Y}-1}{2 \nu_{Y}-3} \tau_{\mathcal{L}} .
$$

If $\nu_{Y} \rightarrow 3^{+}, \tau_{\mathcal{L}}$ converges to $2 \tau_{Y}$, while for $\nu_{Y} \rightarrow+\infty$ we have that $\tau_{\mathcal{L}}$ goes to $\tau_{Y}$. The time scale $\tau_{\sim \mathcal{L}}$ is strictly smaller than $\tau_{\mathcal{L}}$; however, we are implicitly assuming that the characteristic time of the $Z$ process accounts for the volatility persistence, that is $\tau_{Z} \gg \tau_{\mathcal{L}}$, implying that $\tau_{\sim \mathcal{L}}$ is expected to be only slightly smaller than the leverage scale. Ultimately, if $\nu_{Y} \rightarrow 3^{+}, \tau_{<}$converges to $2 \tau_{\mathcal{L}} / 3$, while under the Gaussian limit we have that $\tau_{<}$converges to $\tau_{\mathcal{L}} / 2$. In fact, the three leverage scales are constrained in a narrow range, which empirically has been found to be of order ten days for indexes, or even larger for single stocks 22 .

\subsection{Autocorrelation function of squared increments}

The volatility clustering is commonly measured by the quantity $\mathbb{E}\left[\mathrm{d} X_{t}^{2} \mathrm{~d} X_{t+\tau}^{2}\right]$ and the volatility autocorrelation can be estimated in terms of the following normalized quantity

$$
\mathcal{A}(\tau ; t)=\frac{\mathbb{E}\left[d X_{t}^{2} d X_{t+\tau}^{2}\right]-\mathbb{E}\left[d X_{t}^{2}\right] \mathbb{E}\left[d X_{t+\tau}^{2}\right]}{\sqrt{\operatorname{Var}\left[d X_{t}^{2}\right] \operatorname{Var}\left[d X_{t+\tau}^{2}\right]}} .
$$

To complete our analytical characterization of the two-factor model (4), in Appendix C we derive the explicit expression of (6). At variance with the one previously found in [16 and which is unable to capture the persistence of the volatility, the expression (C.7) features five different exponential scales $\tau_{\mathcal{A}}^{(i=1, \ldots, 5)}$. Similarly to the leverage, the characteristic times are organized in a hierarchy as follows

$$
\begin{aligned}
\tau_{\mathcal{A}}^{(1)} & =-\frac{1}{F_{2}^{Z}}=\frac{\tau_{\mathcal{L}}}{\tau_{Y}} \tau_{Z}=\tau_{>Z}>\tau_{Z}, \\
\tau_{\mathcal{A}}^{(2)} & =-\frac{1}{F_{1}^{Z}}=\tau_{Z}, \\
\tau_{\mathcal{A}}^{(3)} & =-\frac{1}{F_{1}^{Y}}=\tau_{Y}<\tau_{\mathcal{L}}, \\
\tau_{\mathcal{A}}^{(4)} & =-\frac{1}{\left(F_{1}^{Y}+F_{1}^{Z}\right)}=\frac{\tau_{Z}}{\tau_{Z}+\tau_{Y}} \tau_{Y}<\tau_{Y}, \\
\tau_{\mathcal{A}}^{(5)} & =-\frac{1}{F_{2}^{Y}}=\frac{\tau_{\mathcal{L}}}{2} .
\end{aligned}
$$

For $\nu_{Y}$ varying in $(4,+\infty), \tau_{Y}$ is inferiorly bounded by $2 \tau_{\mathcal{L}} / 3$, while the upper bound is given by $\tau_{\mathcal{L}}$. Therefore $\tau_{>Z}$ ranges between $\tau_{Z}$ and $3 \tau_{Z} / 2$, and we can conclude that the previous five scales indeed cluster into two groups, a long-range and a short-range one: the first set is $\left\{\tau_{Z}, \tau_{>Z}\right\}$, whose typical scale is given by $\tau_{Z}$, while the second one contains the three remaining scales, superiorly bounded by $\tau_{\mathcal{L}}$ and of order $\tau_{Y}$. Ultimately, we can appreciate the very reason why model (4) has been enriched by a second factor process $Z_{t}$ w.r.t. the one proposed and discussed in [16. Through the coupling provided by $\rho_{X Y} \neq 0, Y_{t}$ is entirely responsible for the emergence of the leverage; conversely, the Brownian motion driving $Z_{t}$ is decoupled from $W_{t}^{X}$, can not interfere with the leverage, and can not constrain its hierarchy of time scales, as it happens in [16. In model (4) $Z_{t}$ provides the degree of freedom required to capture the persistence of volatility. It is not difficult to imagine that extra volatility factors would 
induce a new plethora of time scales. However, even though the analytical tractability would be preserved, this would come at the cost of an overwhelming burden of messy calculations.

\section{Calibration via Generalized Method of Moments}

In this Section, we propose and discuss an application of the Generalized Method of Moments to the estimation of the model's parameters from en empirical time series of price increments.

The stochastic model (4) is characterized by twelve free parameters, $\tau_{Y}, y_{\infty}, y_{0}$, $\sigma_{Y}^{2}, \tau_{Z}, z_{\infty}, z_{0}, \sigma_{Z}^{2}, \rho_{X Y}, \rho_{X Z}, \rho_{Y Z}$, and $\mu$. Estimating parameters in a stochastic volatility model is a challenging task. This is primarily due to the latency of the volatility state variable. Indeed, in different approaches to volatility modelling, like $\mathrm{ARCH}$ and GARCH models, the likelihood function is readily available. This problem has inspired many scholars, and there is a specialized literature on computationally intensive methods mimicking likelihood-based inference. In general, these belong to the class of non linear filtering methods, and among possible approaches we mention Kalman filters, Particle filters, and Monte Carlo Markov Chain approaches. For more techniques and further discussion we refer the reader to the handbook [19]. Here we take the opportunity to quote the interesting proposal discussed in 31 and rooted on the spectral approach to nonlinear filtering. A relatively simpler approach to estimation, which does not rely on any ad hoc approximation of the density of returns, is based on the computable moments of the model. For continuous-time stochastic volatility models, it is generally very hard to derive closed form solutions for the return moments, but this is not the case for the model under consideration. For this reason, we follow a methodology inspired by the Generalized Method of Moments (GMM). An introduction to the GMM, based on Hansen's formulation of the estimation problem [32, is provided by [33] in Chapter 14. Given $T$ observations $\left\{\mathbf{W}_{t}\right\}$ for $t=1, \ldots, T$, each one being an $h$ dimensional vector, and a vector $\boldsymbol{\theta} \in \mathbb{R}^{k}$ of unknown parameters, in order to apply GMM there should be a function $\mathbf{h}\left(\boldsymbol{\theta}, \mathbf{W}_{t}\right): \mathbb{R}^{k} \times \mathbb{R}^{h} \rightarrow \mathbb{R}^{r}$ characterized by the property that

$$
\mathbb{E}\left[\mathbf{h}\left(\boldsymbol{\theta}, \mathbf{W}_{t}\right)\right]=\mathbf{0} .
$$

These $r$ equalities are usually described as orthogonality conditions. The basic idea of GMM is to replace these conditions with sample averages and to solve the following optimization problem

$$
\hat{\boldsymbol{\theta}}=\underset{\boldsymbol{\theta} \in \boldsymbol{\Theta}}{\operatorname{argmin}}\left(\frac{1}{T} \sum_{t=1}^{T} \mathbf{h}\left(\boldsymbol{\theta}, \mathbf{W}_{t}\right)\right)^{\mathrm{t}} \hat{\boldsymbol{\Omega}}_{T}^{-1}\left(\frac{1}{T} \sum_{t=1}^{T} \mathbf{h}\left(\boldsymbol{\theta}, \mathbf{W}_{t}\right)\right),
$$

where $\hat{\boldsymbol{\Omega}}_{T}$ is a positive-definite weighting matrix depending on the available data set and on the value of $\boldsymbol{\theta}$ itself. The practical procedure is the one which follows. An initial estimate $\hat{\boldsymbol{\theta}}^{(0)}$ is obtained by minimizing the previous quantity with an arbitrary choice of $\hat{\boldsymbol{\Omega}}_{T}$, e.g. $\hat{\boldsymbol{\Omega}}_{T}=\mathbb{I}_{r \times r}$. Supposing that $\mathbf{h}\left(\boldsymbol{\theta}, \mathbf{W}_{t}\right)$ is serially uncorrelated, the estimate $\hat{\boldsymbol{\theta}}^{(0)}$ is then used in

$$
\hat{\boldsymbol{\Omega}}_{T}=\frac{1}{T} \sum_{t=1}^{T} \mathbf{h}\left(\hat{\boldsymbol{\theta}}^{(0)}, \mathbf{W}_{t}\right) \mathbf{h}^{\mathrm{t}}\left(\hat{\boldsymbol{\theta}}^{(0)}, \mathbf{W}_{t}\right)
$$


to arrive to a new GMM estimate $\hat{\boldsymbol{\theta}}^{(1)}$. This process can be iterated until an arbitrary stopping criterion is invoked §. If $\overline{\boldsymbol{\theta}}$ denotes the true value of $\boldsymbol{\theta}$, the theory behind the GMM states that $\hat{\boldsymbol{\theta}}^{(1)}$ is approximately distributed as $\operatorname{Normal}\left(\overline{\boldsymbol{\theta}}, \hat{\boldsymbol{V}}_{T} / T\right)$ with

$$
\hat{\boldsymbol{V}}_{T}=\left\{\left.\left.\frac{\partial}{\partial \boldsymbol{\theta}}\left(\frac{1}{T} \sum_{t=1}^{T} \mathbf{h}_{(k \times r)}^{\mathrm{t}}\left(\boldsymbol{\theta}, \mathbf{W}_{t}\right)\right)\right|_{\boldsymbol{\theta}=\hat{\boldsymbol{\theta}}^{(1)}} \hat{\boldsymbol{\Omega}}_{T}^{-1} \frac{\partial}{\partial \boldsymbol{\theta}^{\mathrm{t}}}\left(\frac{1}{T} \sum_{t=1}^{T} \mathbf{h}\left(\boldsymbol{\theta}, \mathbf{W}_{t}\right)\right)\right|_{\boldsymbol{\theta}=\hat{\boldsymbol{\theta}}^{(1)}}\right\}^{-1} .
$$

For the case under consideration we have $\boldsymbol{\theta}^{\mathrm{t}}=\left(\mu, \tau_{Y}, y_{\infty}, y_{0}, \sigma_{Y}^{2}, \tau_{Z}, z_{\infty}, z_{0}, \sigma_{Z}^{2}, \rho_{X Y}, \rho_{X Z}, \rho_{Y Z}\right)$, while the orthogonality conditions can be obtained computing the lowest order moments of returns, the leverage correlation, and the squared return autocorrelation

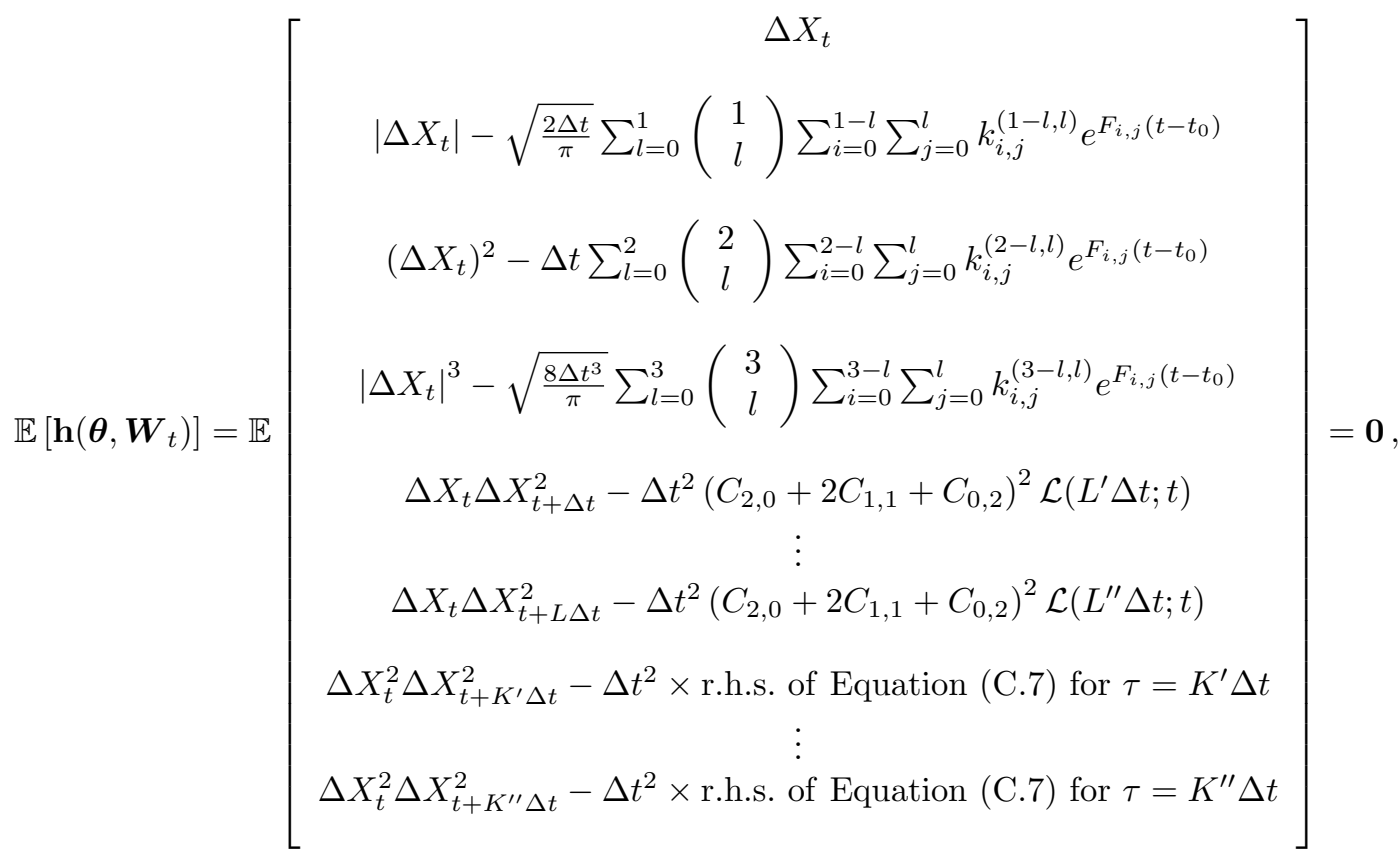

where $\Delta X_{t}=\ln S_{t+\Delta t}-\ln S_{t}-\mu \Delta t, \Delta t=1 / 250$ yr. In the previous equation, the conditions referring to the return-volatility and the squared return correlations depend on the four positive integers $L^{\prime}<L^{\prime \prime}, K^{\prime}<K^{\prime \prime}$. The choice of these values can be made based on a prior analysis of the time scales of the correlation functions, and will be detailed later on. Thus, the dimension $r$ of the vector $\mathbf{h}\left(\boldsymbol{\theta}, \boldsymbol{W}_{t}\right)$ reduces to $4+L^{\prime \prime}-L^{\prime}+1+K^{\prime \prime}-K^{\prime}+1$. From an econometric point of view the problem of the estimation of parameters is cast into a sound statistical framework. By means of GMM we can obtain an estimate of central values and associated statistical uncertainty for all the unknowns of the problem. However, the quantity to be optimized is highly non linear, the optimization procedure of the twelve dimensional problem is per se problematic, and finding a solution under blind search can be extremely demanding. For this reason, we prefer to proceed by invoking some reasonable arguments concerning the nature of the problem under study. The starting point of our

$\S$ When the process $\mathbf{h}\left(\boldsymbol{\theta}, \mathbf{W}_{t}\right)$ for $t=1, \ldots, T$ is serially correlated, the Newey-West estimate for $\hat{\boldsymbol{\Omega}}_{T}$ can be used, please refer to equation 14.1.19 in 33 for further details. 
heuristic is the observation that, until now, we have devoted little attention to the role played by the parameter $t_{0}$. In principle it could be treated as an unknown parameter, however its role is quite different from that played by the others. Since it mainly determines the regime of the factors processes, we assume $t_{0} \rightarrow-\infty$ as done in the previous work [16. Said differently, we assume that the data we are observing reflect stationary realizations of $Y_{t}$ and $Z_{t}$. Under this regime, mean-reverting processes do not depend on the initial time values $y_{0}$ and $z_{0}$ any more, and we identify $y_{\infty}$ with $y_{0}$, and $z_{\infty}$ with $z_{0}$. Moreover, both $Y_{t}$ and $Z_{t}$ are unobserved processes reflecting the presence in the market of investment strategies with heterogeneous time horizons. Even though this assumption could be relaxed, it is plausible to assume that the Brownian motions driving those processes are uncorrelated. If we fix $\rho_{Y Z}=0$ the problem greatly simplifies since all $F_{m, n}$ reduce to $F_{m}^{Y}+F_{n}^{Z}$, and all terms $C_{m, n}$ split into $C_{m, 0} \times C_{0, n}$. In [16] we prove that $\rho_{X Y}<0$ suffices in order to reproduce the leverage effect. Since we do not want that the incorporation of the extra factor $Z_{t}$ has a relevant impact on the leverage, we fix $\rho_{X Z}$ equal to zero. Finally, the considerations that follow Equation (5) in Section 2 have clarified the way the tail exponent of the distribution of the volatility factors is responsible for the divergence of the moments of $X_{t}$. If $Y_{t}$ and $Z_{t}$ were characterized by two different tail exponents, the order of the first divergent moment of $X_{t}$ should be determined by the lowest of them. In this respect the role played by the highest exponent would be spoiled by the other one. We therefore assume that the stationary distributions of $Y_{t}$ and $Z_{t}$ have the same shape parameter $\nu=\nu_{Y}=\nu_{Z}$. Now the reduced vector of parameters reads $\boldsymbol{\theta}^{\mathrm{t}}=\left(\mu, y_{\infty}, z_{\infty}, \tau_{Y}, \tau_{Z}, \rho_{X Y}, \nu\right)$, while the orthogonality relations simplify. For instance, the first four relations reduce to

$$
\begin{aligned}
& \mathbb{E}\left[\Delta X_{t}\right]=0, \\
& \mathbb{E}\left[\left|\Delta X_{t}\right|-\sqrt{\frac{2 \Delta t}{\pi}}\left(y_{\infty}+z_{\infty}\right)\right]=0, \\
& \mathbb{E}\left[\left(\Delta X_{t}\right)^{2}-\left(y_{\infty}+z_{\infty}\right)^{2} \Delta t+\frac{y_{\infty}^{2}+z_{\infty}^{2}}{\nu-2} \Delta t\right]=0, \\
& \mathbb{E}\left[|\Delta X|^{3}-\sqrt{\frac{8 \Delta t^{3}}{\pi}} \frac{(\nu-1)^{2}}{(\nu-3)(\nu-2)}\left(y_{\infty}^{3}+z_{\infty}^{3}\right)\right] \\
& \quad-\mathbb{E}\left[3 \sqrt{\frac{8 \Delta t^{3}}{\pi}} \frac{\nu-1}{\nu-2}\left(y_{\infty}+z_{\infty}\right) y_{\infty} z_{\infty}\right]=0,
\end{aligned}
$$

and the numerator of the leverage for positive $\tau$ becomes

$$
\begin{aligned}
& \rho_{X Y} \sqrt{\frac{8}{\tau_{Y}(\nu-1)}}\left\{\left[C_{2,0}^{\mathrm{st}} C_{0,1}^{\mathrm{st}}-\frac{\nu-1}{\nu-3} y_{\infty} C_{1,0}^{\mathrm{st}} C_{0,1}^{\mathrm{st}}\right] \exp \left[-\left(1+\frac{\nu-2}{\nu-1}\right) \frac{\tau}{\tau_{\mathcal{L}}}\right]\right. \\
& +\left[C_{2,0}^{\mathrm{st}} C_{0,1}^{\mathrm{st}}+C_{1,0}^{\mathrm{st}} C_{0,2}^{\mathrm{st}}-z_{\infty}\left(C_{2,0}^{\mathrm{st}}+C_{1,0}^{\mathrm{st}} C_{0,1}^{\mathrm{st}}\right)\right] \exp \left[-\left(1+\frac{\tau_{\mathcal{L}}}{\tau_{Z}}\right) \frac{\tau}{\tau_{\mathcal{L}}}\right] \\
& \left.+\left[\left(\frac{\nu-1}{\nu-3} y_{\infty}+z_{\infty}\right)\left(C_{2,0}^{\mathrm{st}}+C_{1,0}^{\mathrm{st}} C_{0,1}^{\mathrm{st}}\right)\right] \exp \left(-\frac{\tau}{\tau_{\mathcal{L}}}\right)\right\},
\end{aligned}
$$

where the superscript ${ }^{\text {st }}$ stands for the stationary regime corresponding to $t_{0} \rightarrow-\infty$, and we recall that $\tau_{\mathcal{L}}=(\nu-1) \tau_{Y} /(\nu-2)$. Even though the leverage correlation introduces a superposition of three exponential functions, we have seen at the end of the Section 3.1 that the characteristic exponents are of the same magnitude and 
are all dominated by $\tau_{\mathcal{L}}$. For this reason, and recalling that the typical decay time for the leverage is smaller than one hundred days, to perform the optimization we fix $L^{\prime}=1, L^{\prime \prime}=250$, and solve the problem for the first 254 orthogonal relations. This greatly enhances the convergence of the numerical algorithms. Once an estimate of $\tau_{\mathcal{L}}$ is found, we fix $K^{\prime}$ equal to two times the integer part of $\tau_{\mathcal{L}}, K^{\prime}=\left\lfloor 2 \tau_{\mathcal{L}}\right\rfloor|\mathbb{\|}|$ and $K^{\prime \prime}=250$, and we perform the final optimization on the entire set of $254+K^{\prime \prime}-\bar{K}^{\prime}+1$ orthogonal relations. The latter relations, which correspond to the volatility structure, should be fixed using the whole range for the lagged correlation, and not just the lags above the leverage. However, we preliminarily perform the numerical optimization with $K^{\prime}$ running from one to the mentioned level and we see that the fit worsens. The reason comes from the very structure of the expression which appears in the r.h.s. of Equation (C.7). As commented in Section 3.2, the lagged correlation is dominated by two time scales, the first one is of order of $\tau_{\mathcal{L}}$, while the second one is the long run component. When $K^{\prime}=1$, we are fitting the behaviour of the whole curve, but for low lags it is largely determined by the scaling of the leverage. The results of our numerical explorations show that the low lags part of the curve is hard to be reproduced while the long run component is always well described. When we reduce $K^{\prime}$ to one the optimizer tries to catch up at short lags but at the cost of an even worst distortion of the autocorrelation for intermediate lag values, please refer to Figure 2 for a visual comparison of the cases $K^{\prime}=1$ and $K^{\prime}=\left\lfloor 2 \tau_{\mathcal{L}}\right\rfloor$. In light of these results we decide to perform the GMM favouring the long run behaviour. An alternative and less abrupt approach could be a weighted optimization on the entire curve with weights in the matrix $\hat{\boldsymbol{\Omega}}_{T}$ that change smoothly from zero to one from low to high lag values. Finally we perform the GMM just one time and we obtain $\hat{\boldsymbol{\theta}}^{(1)}$ and $\hat{\boldsymbol{V}}_{T} / T$. In order to compute a consistent estimate of $\sigma_{Y}^{2}$ and the associated confidence level, we can extract a random sample from $\operatorname{Normal}\left(\hat{\boldsymbol{\theta}}^{(1)}, \hat{\boldsymbol{V}}_{T} / T\right)$ and obtain a statistics of $\sigma_{Y}^{2}$ through the relation $2 /\left(\tau_{Y}(\nu-1)\right)$. We proceed in an analogous way for $\sigma_{Z}^{2}$, and for $\tau_{\mathcal{L}}=\tau_{Y}(\nu-1) /(\nu-2)$. The time series on which we perform the analysis is the same used in [16, and it consists of a data set from the Standard \& Poors 500 index daily returns from 1970 to 2010. This allows to evaluate the ability of the extended model to capture the persistence of the volatility, not only in absolute terms but also in comparison with the previous estimate from a simpler model. In Table 1 we report the central values $\hat{\boldsymbol{\theta}}^{(1)}$, the standard errors $\hat{\boldsymbol{\sigma}}_{T}=\sqrt{\operatorname{diag}\left(\hat{\mathbf{V}}_{T} / T\right)}$, and the correlation structure $\hat{\boldsymbol{\rho}}_{T}=\hat{\mathbf{V}}_{T} /\left(T \hat{\boldsymbol{\sigma}}_{T} \hat{\boldsymbol{\sigma}}_{T}^{\mathrm{t}}\right)$ for all the parameters. As far as the other relevant parameters of the model are concerned, we have $\sigma_{Y}^{2}=9.9 \pm 1.9, \sigma_{Z}^{2}=1.58 \pm 0.07$, and $\tau_{\mathcal{L}}=0.10 \pm 0.02 \mathrm{yr}$. The new values confirm the goodness of the estimate provided in [16, in particular the value of $\rho_{X Y}$ is strictly negative and the level of the tail exponent $\nu$ predicts the divergence of moments higher than the fourth one. More interesting to comment is the relationship between the different time scales involved in our process. Indeed, the shortest time scale corresponds to the typical relaxation time of $Y_{t}$, which is found to be equal to $0.07 \pm 0.01 \mathrm{yr}$ and is therefore dominated by the leverage time scale $0.10 \pm 0.02 \mathrm{yr}$ (to be compared with the old estimate for $\tau_{\mathcal{L}}$ in [16] which is $0.09 \mathrm{yr}$ ). The new time scale $\tau_{Z}$ for the process $Z_{t}$ is found to be a factor of six larger than that of $Y_{t}$. In Figures 1 and 2 we plot the leverage function

\|I In the following we adopt standard mathematical notation $\lfloor\cdot\rfloor$ for the integer part function. 
Figure 1. Analytical description of the empirical leverage correlation with values of parameters estimated by GMM with $K^{\prime}=\left\lfloor 2 \tau_{\mathcal{L}}\right\rfloor$.

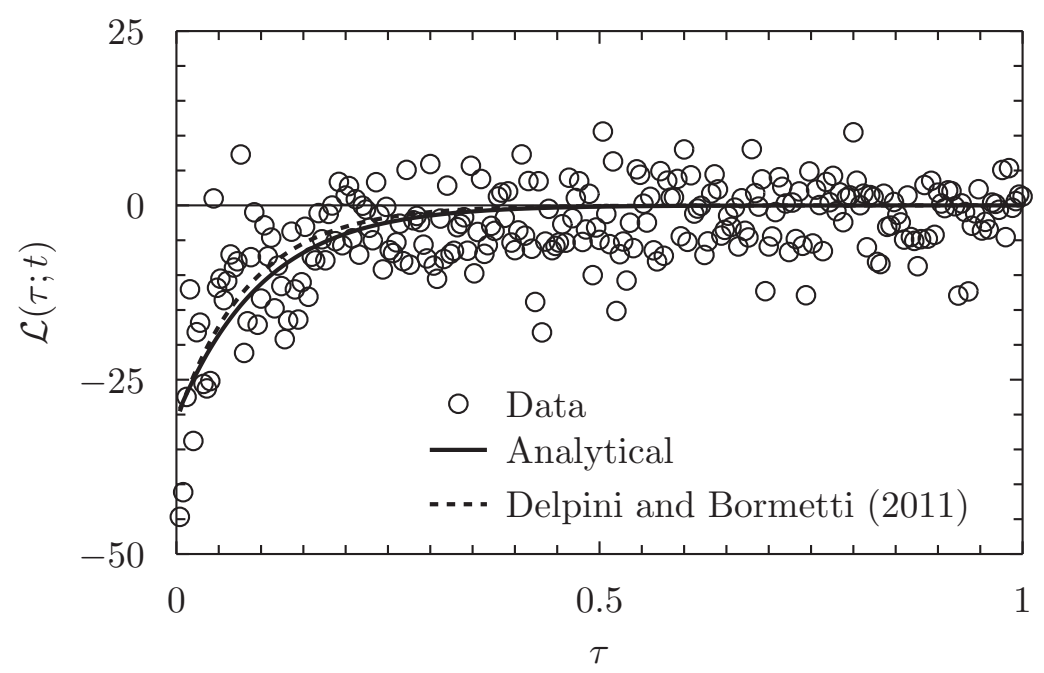

Figure 2. Empirical volatility autocorrelation function of the daily returns of the S\&P500 index 1970-2010 (data points), and analytical descriptions: bold and dotted lines, new expressions with GMM estimates for different values of $K^{\prime}$; dashed line, formula and values of parameters as in 16 .

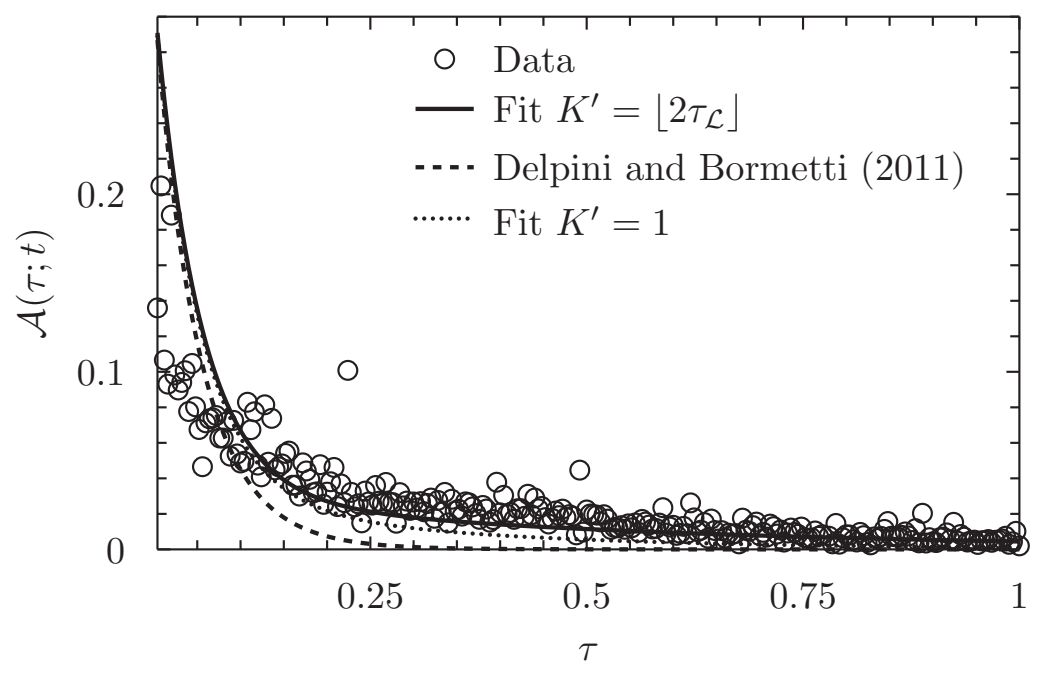

and the normalized autocorrelation of squared returns. The exponential decay of the leverage is described correctly by the analytical formula, and no relevant differences are noticeable with respect to the description obtained via the model introduced in [16]. Different considerations apply to the persistence of the volatility as predicted by the extended model. The presence of the slow volatility factor $Z_{t}$ introduces a longer time scale allowing to capture the long range memory of the autocorrelation function. 
Table 1. Estimated values of the parameters $\left(K^{\prime}=\left\lfloor 2 \tau_{\mathcal{L}}\right\rfloor\right)$ from daily returns of the S\&P500 index 1970-2010 .

\begin{tabular}{|c|c|c|c|c|c|c|c|c|c|}
\hline & $\hat{\boldsymbol{\theta}}^{(1)}$ & $\hat{\boldsymbol{\sigma}}_{T}$ & & & & $\hat{\boldsymbol{\rho}}_{T}$ & & & \\
\hline$\mu$ & $2.1 \times 10^{-4}$ & $6 \times 10^{-5}$ & 1.00 & -0.01 & 0.02 & -0.28 & -0.01 & -0.01 & -0.01 \\
\hline$y_{\infty}$ & 0.095 & 0.004 & -0.01 & 1.00 & -0.97 & -0.04 & -0.14 & 0.00 & 0.99 \\
\hline$z_{\infty}$ & 0.052 & 0.004 & 0.02 & -0.97 & 1.00 & 0.03 & 0.25 & 0.00 & -0.94 \\
\hline$\tau_{Y}$ & $0.07 \mathrm{yr}$ & $0.01 \mathrm{yr}$ & -0.28 & -0.04 & 0.03 & 1.00 & 0.05 & 0.01 & -0.05 \\
\hline$\tau_{Z}$ & $0.40 \mathrm{yr}$ & $0.02 \mathrm{yr}$ & -0.01 & -0.14 & 0.25 & 0.05 & 1.00 & 0.00 & -0.12 \\
\hline$\rho_{X Y}$ & -0.77 & 0.09 & -0.01 & 0.00 & 0.00 & 0.01 & 0.00 & 1.00 & 0.00 \\
\hline$\nu$ & 4.15 & 0.01 & -0.01 & 0.99 & -0.94 & -0.05 & -0.12 & 0.00 & 1.00 \\
\hline
\end{tabular}

This is evident from the comparison between the dashed line, corresponding to the old model, and the bold one, corresponding to model (4). Our results demonstrate the ability of a multi-factor approach to stochastic volatility to effectively describe several phenomena. In particular, even in the simplest version of a two factor model, it is able to capture the emergence of multiple time scales for the volatility autocorrelation as well as the exponential decay of the return-volatility correlation. The measured value for the tail parameter $\nu$ is coherent with the internal consistency of the model requiring $\nu$ to be greater than four (in order for Equation (C.7) to converge in the stationary limit). In particular, $\nu=4.15$ predicts an hyperbolic decay of the daily return distribution which captures correctly the non Gaussian probability of extreme events in the real data.

\section{Conclusions}

In this work the model for the description of financial stylized facts proposed in 16] is amended from the unrealistic fast decay of the volatility autocorrelation. This is achieved introducing an extra stochastic factor driving the volatility. In principle the number of factors could be increased at will, but the analytical tractability of the resulting model would be hardly exploitable. The intuition behind this generalization traces back to the early empirical analysis of the FX market in 9 and the model in [10, where the role played by heterogeneous investors is strongly emphasized. Evidences from these papers are rooted in the econometric analysis of publicly available financial time series, but a convincing micro-founded model is still lacking. Access to electronic order book data and to agents' identifiers would allow to estimate the individual components of this heterogeneity. An even approximate estimation of the distribution of typical investment horizons from this information would provide a valuable traderbased foundation.

With respect to previous approaches and analyses of continuous-time stochastic volatility models, we believe that the calibration procedure proposed here represents a further improvement and fulfils the desirable requirements of statistical soundness. At the same time, it also allows to focus on those facts which are established as relevant for the description of financial data. In particular, we pursue an heuristic approach to 
optimization that, reducing the dimensionality of the parameters space, retains only those ingredients which are actually needed to capture the aforementioned empirical evidences.

The stochastic volatility models discussed in this paper and in [16] are inspired exclusively by the quest for a realistic description of financial data. In this quest, we focus on continuous time modelling and do not consider aspects relating to possible applications in the financial sector. In this respect, we should mention that important progresses have been obtained by discrete time models. In particular, models with multiscale ARCH volatility can also accommodate many stylized facts, like the fat tails of returns, and reproduce consistently the dynamics of realized volatility, also delivering accurate forecasts of the latter [34. They also prove to be very flexible tools for efficient option pricing and hedging purposes [35].

In our case, the emergence of power-law tails in the return distribution complies with past empirical analysis [36, but also poses serious limitations to the usage of the model in the context of option pricing. This is certainly true for vanilla instruments, whose payoff grows exponentially with the log-price. On the other hand, our framework could deliver, in principle, better estimates of the role played by rare events for market risk evaluation.

On the whole, we believe our model achieves a remarkable degree of realism, higher than previous attempts in continuous-time stochastic volatility modelling, yet allowing for important analytical derivations, e.g. that of the moments of the return probability distribution. Fairly enough, this comes at the price of elaborate manipulations and Monte Carlo simulation would still be due for most financially relevant applications. Nonetheless recent advances in fast computing, e.g. GPU based numerical techniques, could offer a promising scenario in this regard.

\section{Acknowledgments}

We thank the anonymous referees for constructive comments and suggestions. We also warmly thank Fulvio Corsi for many inspiring discussions and we both acknowledge support of the Scuola Normale Superiore Grant 'Giovani Ricercatori 2011'.

\section{Appendix A. Probability density function of price returns: moments computation}

Application of Itô's Lemma to the function $X_{t}^{l}$ readily provides

$$
\mathbb{E}\left[X_{t}^{l}\right]=\frac{1}{2} l(l-1) \int_{0}^{t} \mathbb{E}\left[X_{s}^{l-2}\left(Y_{s}+Z_{s}\right)^{2}\right] \mathrm{d} s,
$$

and the same Lemma proves that the correlation functions between integer powers of $X_{t}, Y_{t}$, and $Z_{t}$ satisfy the following differential equation

$$
\begin{aligned}
& \frac{\mathrm{d}}{\mathrm{d} t} \mathbb{E}\left[X_{t}^{l} Y_{t}^{m} Z_{t}^{n}\right]=F_{m, n} \mathbb{E}\left[X_{t}^{l} Y_{t}^{m} Z_{t}^{n}\right]+A_{m}^{Y} \mathbb{E}\left[X_{t}^{l} Y_{t}^{m-1} Z_{t}^{n}\right] \\
& +A_{n}^{Z} \mathbb{E}\left[X_{t}^{l} Y_{t}^{m} Z_{t}^{n-1}\right]+\frac{1}{2} l(l-1) \mathbb{E}\left[X_{t}^{l-2} Y_{t}^{m} Z_{t}^{n}\left(Y_{t}+Z_{t}\right)^{2}\right] \\
& +l\left(m \rho_{X Y} \sigma_{Y}+n \rho_{X Z} \sigma_{Z}\right) \mathbb{E}\left[X_{t}^{l-1} Y_{t}^{m} Z_{t}^{n}\left(Y_{t}+Z_{t}\right)\right],
\end{aligned}
$$


where the constants $F_{m, n}$ are defined right after Equation (5) and $A_{m}^{Y}=m \kappa_{Y} y_{\infty}$ and $A_{n}^{Z}=n \kappa_{Z} z_{\infty}$. Previous equations correspond to a system of nested linear ordinary differential equation (ODE), which can be solved recursively starting from the lowest order of $l, m$, and $n$, and whose solution involves integration of the two point correlations $C_{m, n}\left(t ; t_{0}\right)=\mathbb{E}\left[Y_{t}^{m} Z_{t}^{n}\right] \mathbb{\mathbb { T }}$. From application of Itô's Lemma we obtain

$$
\begin{aligned}
& \mathrm{d}\left(Y^{m} Z^{n}\right)=\left[F_{m}^{Y} Y^{m} Z^{n}+F_{n}^{Z} Y^{m} Z^{n}\right] \mathrm{d} t \\
& +\left[A_{m}^{Y} Y^{m-1} Z^{n}+A_{n}^{Z} Y^{m} Z^{n-1}\right] \mathrm{d} t+\rho_{Y Z} m n \sqrt{\sigma_{Y}^{2} \sigma_{Z}^{2}} Y^{m} Z^{n} \mathrm{~d} t \\
& +m \sqrt{\sigma_{Y}^{2}} Y^{m} Z^{n} \mathrm{~d} W_{t}^{Y}+n \sqrt{\sigma_{Z}^{2}} Y^{m} Z^{n} \mathrm{~d} W_{t}^{Z} ;
\end{aligned}
$$

taking expectation, and differentiating w.r.t time we derive the following ODE

$$
\begin{aligned}
& \frac{\mathrm{d}}{\mathrm{d} t} \mathbb{E}\left[Y_{t}^{m} Z_{t}^{n}\right]=\left(F_{m}^{Y}+F_{n}^{Z}+\rho_{Y Z} m n \sqrt{\sigma_{Y}^{2} \sigma_{Z}^{2}}\right) \mathbb{E}\left[Y_{t}^{m} Z_{t}^{n}\right] \\
& +A_{m}^{Y} \mathbb{E}\left[Y_{t}^{m-1} Z_{t}^{n}\right]+A_{n}^{Z} \mathbb{E}\left[Y_{t}^{m} Z_{t}^{n-1}\right] .
\end{aligned}
$$

For instance, for the case $m=n=1$ we have

$$
\begin{aligned}
& \frac{\mathrm{d}}{\mathrm{d} t} \mathbb{E}\left[Y_{t} Z_{t}\right]=\left(F_{1}^{Y}+F_{1}^{Z}+\rho_{Y Z} \sqrt{\sigma_{Y}^{2} \sigma_{Z}^{2}}\right) \mathbb{E}\left[Y_{t} Z_{t}\right] \\
& +A_{1}^{Y} \mathbb{E}\left[Z_{t}\right]+A_{1}^{Z} \mathbb{E}\left[Y_{t}\right],
\end{aligned}
$$

where the mean values read

$$
\begin{aligned}
& \mathbb{E}\left[Y_{t}\right]=-\frac{A_{1}^{Y}}{F_{1}^{Y}}+e^{F_{1}^{Y}\left(t-t_{0}\right)}\left[y_{0}+\frac{A_{1}^{Y}}{F_{1}^{Y}}\right] \\
& \mathbb{E}\left[Z_{t}\right]=-\frac{A_{1}^{Z}}{F_{1}^{Z}}+e^{F_{1}^{Z}\left(t-t_{0}\right)}\left[z_{0}+\frac{A_{1}^{Z}}{F_{1}^{Z}}\right],
\end{aligned}
$$

with $t_{0} \leq 0$ the starting time of the factors processes. More generally, by iterative solution it can be verified that $C_{m, n}$ admits the expansion

$$
C_{m, n}=\mathbb{E}\left[Y_{t}^{m} Z_{t}^{n}\right]=\sum_{i=0}^{m} \sum_{j=0}^{n} k_{i, j}^{(m, n)} e^{F_{i, j}\left(t-t_{0}\right)},
$$

where the coefficients depend on the initial conditions $y_{0}, z_{0}$ and satisfy the recursive relations which follow

$$
\begin{aligned}
k_{i<m, j<n}^{(m, n)}= & -\frac{A_{m}^{Y} k_{i, j}^{(m-1, n)}+A_{n}^{Z} k_{i, j}^{(m, n-1)}}{F_{m, n}-F_{i, j}} \\
k_{i<m, n}^{(m, n)}= & -\frac{A_{m}^{Y} k_{i, n}^{(m-1, n)}}{F_{m, n}-F_{i, n}} \\
k_{m, j<n}^{(m, n)}= & -\frac{A_{n}^{Z} k_{m, j}^{(m, n-1)}}{F_{m, n}-F_{m, j}} \\
k_{m, n}^{(m, n)}= & \mathbb{E}\left[Y_{t_{0}}^{m} Z_{t_{0}}^{n}\right]+A_{m}^{Y} \sum_{i=0}^{m-1} \sum_{j=0}^{n} \frac{k_{i, j}^{(m-1, n)}}{F_{m, n}-F_{i, j}} \\
& +A_{n}^{Z} \sum_{i=0}^{m} \sum_{j=0}^{n-1} \frac{k_{i, j}^{(m, n-1)}}{F_{m, n}-F_{i, j}} .
\end{aligned}
$$

I In the following we drop the dependence on $t$ and $t_{0}$. 
We notice that the moments $\mu_{m}^{Y}(t)=\mathbb{E}\left[Y_{t}^{m}\right]$ and $\mu_{n}^{Z}(t)=\mathbb{E}\left[Z_{t}^{n}\right]$ are specific cases of the expansion A.3, whose coefficients are given by the column vector $\left(k_{i, 0}^{(m, 0)}\right)_{i \leq m}$ and the row vector $\left(k_{0, j}^{(0, n)}\right)_{j \leq n}$, while in general the set of coefficients $k_{i, j}^{(m, n)}$ can be cast in a $(m+1) \times(n+1)$ real matrix. For instance, for the case $C_{2,1}=\mathbb{E}\left[Y_{t}^{2} Z_{t}\right]$ we obtain

$$
\begin{aligned}
& k_{0,0}^{(2,1)}=-\frac{1}{F_{2,1}}\left[A_{2}^{Y} k_{0,0}^{(1,1)}+A_{1}^{Z} k_{0,0}^{(2,0)}\right], \\
& k_{0,1}^{(2,1)}=-\frac{A_{2}^{Y} k_{0,1}^{(1,1)}}{F_{2,1}-F_{1}^{Z}}, \\
& k_{1,0}^{(2,1)}=-\frac{1}{F_{2,1}-F_{1}^{Y}}\left[A_{2}^{Y} k_{1,0}^{(1,1)}+A_{1}^{Z} k_{1,0}^{(2,0)}\right], \\
& k_{1,1}^{(2,1)}=-\frac{A_{2}^{Y} k_{1,1}^{(1,1)}}{F_{2,1}-F_{1,1}}, \\
& k_{2,0}^{(2,1)}=-\frac{A_{1}^{Z} k_{2,0}^{(2,0)}}{F_{2,1}-F_{2}}, \\
& k_{2,1}^{(2,1)}=\mathbb{E}\left[Y_{t_{0}}^{2} Z_{t_{0}}\right]-\left[k_{0,0}^{(2,1)}+k_{0,1}^{(2,1)}+k_{1,0}^{(2,1)}+k_{1,1}^{(2,1)}+k_{2,0}^{(2,1)}\right] .
\end{aligned}
$$

Ultimately, given the expansion A.3 and by inspection of A.1 , we recognize that the moments of $X$ can be cast in the form of Equation (5).

\section{Appendix B. Computation of the return-volatility correlation}

The results which follow are derived under the assumption that $\rho_{X Z}$ is equal to zero. The numerator of the return-volatility correlation $\mathcal{L}(\tau ; t)=\mathbb{E}\left[\mathrm{d} X_{t} \mathrm{~d} X_{t+\tau}^{2}\right] / \mathbb{E}\left[\mathrm{d} X_{t}^{2}\right]^{2}$ can be cast in the form

$$
\mathbb{E}\left[\mathrm{d} X_{t} \mathrm{~d} X_{t+\tau}^{2}\right]=\mathbb{E}\left[\left(Y_{t}+Z_{t}\right)\left(Y_{t+\tau}+Z_{t+\tau}\right)^{2} \zeta_{t}^{X}\right] \mathrm{d} t^{2} .
$$

Here, adopting the same convention of [25, we formally $\rightarrow$ express the Wiener increment as $\mathrm{d} W_{t}^{X}=\zeta_{t}^{X} \mathrm{~d} t$, where $\zeta_{t}^{X}$ is a Gaussian noise with zero mean and variance $\mathbb{E}\left[\left(\zeta_{t}^{X}\right)^{2}\right]=1 / \mathrm{d} t$. Novikov's theorem [37, 38] allows to compute the expectation involving $\zeta_{t}^{X}$, giving us

$$
\begin{aligned}
& \frac{\mathbb{E}\left[\mathrm{d} X_{t} \mathrm{~d} X_{t+\tau}^{2}\right]}{\mathrm{d} t^{2}}=2 \rho_{X Y} \sqrt{\sigma_{Y}^{2}} H(\tau) \exp \left(-\kappa_{Y} \tau\right) \times \\
& \mathbb{E}\left[\left[Y_{t}\left(Y_{t}+Z_{t}\right)\left(Y_{t+\tau}+Z_{t+\tau}\right)\right] \exp \left[\sqrt{\sigma_{Y}^{2}} \Delta_{t} W^{Y}(\tau)\right]\right],
\end{aligned}
$$

where we define $\Delta_{t} W(\tau) \doteq \int_{t}^{t+\tau} d W_{s}$. We refer the interested reader to Section IV in [16] for further details regarding the derivation of the previous equation. The right hand side of (B.1) can be split into four pieces proportional to the expectations

$$
f_{Y Y Y}(\tau, t) \doteq \mathbb{E}\left[Y_{t}^{2} Y_{t+\tau} \exp \left[\sqrt{\sigma_{Y}^{2}} \Delta_{t} W^{Y}(\tau)\right]\right],
$$

\footnotetext{
+ This convention is somewhat unusual, but admissible as the distributional assumption on $\zeta_{t}^{X}$ guarantees that $d W_{t}$ is still Gaussian with zero mean and variance equal to $\mathrm{d} t$. This choice proves to be convenient to develop the following calculations.
} 
Stochastic volatility with heterogeneous time scales

$$
\begin{aligned}
f_{Y Y Z}(\tau, t) & \doteq \mathbb{E}\left[Y_{t}^{2} Z_{t+\tau} \exp \left[\sqrt{\sigma_{Y}^{2}} \Delta_{t} W^{Y}(\tau)\right]\right], \\
f_{Y Z Y}(\tau, t) & \doteq \mathbb{E}\left[Y_{t} Z_{t} Y_{t+\tau} \exp \left[\sqrt{\sigma_{Y}^{2}} \Delta_{t} W^{Y}(\tau)\right]\right], \\
f_{Y Z Z}(\tau, t) & \doteq \mathbb{E}\left[Y_{t} Z_{t} Z_{t+\tau} \exp \left[\sqrt{\sigma_{Y}^{2}} \Delta_{t} W^{Y}(\tau)\right]\right] .
\end{aligned}
$$

Following the approach discussed in Appendix B of [16], it is possible to show that they satisfy the relations

$$
\begin{aligned}
& f_{Y Y Y}(\tau, t)-\left(\sigma_{Y}^{2}-\kappa_{Y}\right) \int_{0}^{\tau} f_{Y Y Y}\left(\tau^{\prime}, t\right) \exp \left[\frac{\sigma_{Y}^{2}}{2}\left(\tau-\tau^{\prime}\right)\right] \mathrm{d} \tau^{\prime}= \\
& \exp \left(\frac{\sigma_{Y}^{2}}{2} \tau\right)\left[C_{3,0}+\kappa_{Y} y_{\infty} \tau C_{2,0}\right], \\
& f_{Y Y Z}(\tau, t)+\kappa_{Z} \int_{0}^{\tau} f_{Y Y Z}\left(\tau^{\prime}, t\right) \exp \left[\frac{\sigma_{Y}^{2}}{2}\left(\tau-\tau^{\prime}\right)\right] \mathrm{d} \tau^{\prime}= \\
& \exp \left(\frac{\sigma_{Y}^{2}}{2} \tau\right)\left[C_{2,0} C_{0,1}+\kappa_{Z} z_{\infty} \tau C_{2,0}\right], \\
& f_{Y Z Y}(\tau, t)-\left(\sigma_{Y}^{2}-\kappa_{Y}\right) \int_{0}^{\tau} f_{Y Z Y}\left(\tau^{\prime}, t\right) \exp \left[\frac{\sigma_{Y}^{2}}{2}\left(\tau-\tau^{\prime}\right)\right] \mathrm{d} \tau^{\prime}= \\
& \exp \left(\frac{\sigma_{Y}^{2}}{2} \tau\right)\left[C_{2,0} C_{0,1}+\kappa_{Y} y_{\infty} \tau C_{1,0} C_{0,1}\right], \\
& f_{Y Z Z}(\tau, t)+\kappa_{Z} \int_{0}^{\tau} f_{Y Z Z}\left(\tau^{\prime}, t\right) \exp \left[\frac{\sigma_{Y}^{2}}{2}\left(\tau-\tau^{\prime}\right)\right] \mathrm{d} \tau^{\prime}= \\
& \exp \left(\frac{\sigma_{Y}^{2}}{2} \tau\right)\left[C_{1,0} C_{0,2}+\kappa_{Z} z_{\infty} \tau C_{1,0} C_{0,1}\right],
\end{aligned}
$$

corresponding to a set of Volterra integro-differential equations of the second kind. Their solutions are known in closed-form, and after plugging them in Equation (B.1), the final expression of the leverage correlation reads

$$
\begin{aligned}
& \mathcal{L}(\tau ; t)=\frac{2 \rho_{X Y} \sqrt{\sigma_{Y}^{2}} H(\tau)}{\left(C_{2,0}+2 C_{1,0} C_{0,1}+C_{0,2}\right)^{2}} \times \\
& \left\{\left[C_{3,0}+C_{2,0} C_{0,1}+\frac{\kappa_{Y} y_{\infty}}{\sigma_{Y}^{2}-\kappa_{Y}}\left(C_{2,0}+C_{1,0} C_{0,1}\right)\right] \exp \left[2\left(\frac{3 \sigma_{Y}^{2}}{4}-\kappa_{Y}\right) \tau\right]\right. \\
& +\left[C_{2,0} C_{0,1}+C_{1,2}-z_{\infty}\left(C_{2,0}+C_{1,0} C_{0,1}\right)\right] \exp \left[\left(\frac{\sigma_{Y}^{2}}{2}-\kappa_{Y}-\kappa_{Z}\right) \tau\right] \\
& \left.-\left[\left(\frac{\kappa_{Y} y_{\infty}}{\sigma_{Y}^{2}-\kappa_{Y}}-z_{\infty}\right)\left(C_{2,0}+C_{1,0} C_{0,1}\right)\right] \exp \left[\left(\frac{\sigma_{Y}^{2}}{2}-\kappa_{Y}\right) \tau\right]\right\} .
\end{aligned}
$$

A meaningful comparison of the previous expression with real data requires to take its stationary limit for $t_{0} \rightarrow-\infty$, which amounts to replacing $C_{m, 0} C_{0, n}$ with the asymptotic values $C_{m, 0}^{\mathrm{st}} C_{0, n}^{\mathrm{st}}$. 


\section{Appendix C. Computation of the squared return correlation}

Resorting to the same parametrization of the Wiener variation adopted in Appendix B] we have

$$
\begin{aligned}
\mathbb{E}\left[\mathrm{d} X_{t}^{2} \mathrm{~d} X_{t+\tau}^{2}\right] & =\mathrm{d} t^{2} \mathbb{E}\left[\left(Y_{t}+Z_{t}\right)^{2}\left(Y_{t+\tau}+Z_{t+\tau}\right)^{2} \mathrm{~d} W_{t}^{X} \zeta_{t}^{X}\right] \\
& =\mathrm{d} t^{2} \mathbb{E}\left[\left(Y_{t}+Z_{t}\right)^{2}\left(Y_{t+\tau}+Z_{t+\tau}\right)^{2}\right]+\mathcal{O}\left(\mathrm{d} t^{3}\right)
\end{aligned}
$$

In order to compute the autocorrelation function of squared returns, the quantities $f_{t}^{(m, n, p, q)}(\tau)=\mathbb{E}\left[Y_{t}^{m} Z_{t}^{n} Y_{t+\tau}^{p} Z_{t+\tau}^{q}\right]$ indicating the $\tau$-lagged correlation have to be evaluated. The relevant cases correspond to $p, q \leq 2$, and below we detail the corresponding exact results, all of which are obtained replacing the process $Y_{t+\tau}^{p} Z_{t+\tau}^{q}$ with its integral representation from time $t$ to time $t+\tau$.

Computation of $f_{t}^{(m, n, 1,0)}(\tau)=\mathbb{E}\left[Y_{t}^{m} Z_{t}^{n} Y_{t+\tau}\right]$. It is readily verified that $f_{t}^{(m, n, 1,0)}(\tau)$ is solution of a linear ODE, giving

$$
f_{t}^{(m, n, 1,0)}(\tau)=-\frac{A_{1}^{Y}}{F_{1}^{Y}} C_{m, n}+e^{F_{1}^{Y} \tau}\left[C_{m+1, n}+\frac{A_{1}^{Y}}{F_{1}^{Y}} C_{m, n}\right] .
$$

Computation of $f_{t}^{(m, n, 0,1)}(\tau)=\mathbb{E}\left[Y_{t}^{m} Z_{t}^{n} Z_{t+\tau}\right]$. In much the same way we have

$$
f_{t}^{(m, n, 0,1)}(\tau)=-\frac{A_{1}^{Z}}{F_{1}^{Z}} C_{m, n}+e^{F_{1}^{Z} \tau}\left[C_{m, n+1}+\frac{A_{1}^{Z}}{F_{1}^{Z}} C_{m, n}\right] \text {. }
$$

Computation of $f_{t}^{(m, n, 2,0)}(\tau)=\mathbb{E}\left[Y_{t}^{m} Z_{t}^{n} Y_{t+\tau}^{2}\right]$. After replacement of $Y_{t+\tau}^{2}$, we can write

$$
\begin{aligned}
f_{t}^{(m, n, 2,0)}(\tau)= & f_{t}^{(m, n, 2,0)}(0)+F_{2}^{Y} \int_{0}^{\tau} f_{t}^{(m, n, 2,0)}\left(\tau^{\prime}\right) \mathrm{d} \tau^{\prime} \\
& +A_{2}^{Y} \int_{0}^{\tau} f_{t}^{(m, n, 1,0)}\left(\tau^{\prime}\right) \mathrm{d} \tau^{\prime}
\end{aligned}
$$

further, we can replace the solution C.2 for $f_{t}^{(m, n, 1,0)}\left(\tau^{\prime}\right)$ in the second integral, leading to straightforward integrations of exponential functions of $\tau$. Finally, we are left with

$$
\begin{aligned}
f_{t}^{(m, n, 2,0)}(\tau) & =\left[\frac{A_{2}^{Y} A_{1}^{Y}}{F_{2}^{Y} F_{1}^{Y}} C_{m, n}\right] \\
+ & e^{F_{1}^{Y} \tau}\left[-\frac{A_{2}^{Y}}{F_{2}^{Y}-F_{1}^{Y}}\left(C_{m+1, n}+\frac{A_{1}^{Y}}{F_{1}^{Y}} C_{m, n}\right)\right] \\
+ & e^{F_{2}^{Y} \tau}\left[C_{m+2, n}+\frac{A_{2}^{Y}}{F_{2}^{Y}-F_{1}^{Y}}\left(C_{m+1, n}+\frac{A_{1}^{Y}}{F_{2}^{Y}} C_{m, n}\right)\right] .
\end{aligned}
$$

Computation of $f_{t}^{(m, n, 0,2)}(\tau)=\mathbb{E}\left[Y_{t}^{m} Z_{t}^{n} Z_{t+\tau}^{2}\right]$. As before, after replacement of the parameters for the dynamics of the $Z_{t+\tau}^{2}$ process, we get to

$$
f_{t}^{(m, n, 0,2)}(\tau)=\left[\frac{A_{2}^{Z} A_{1}^{Z}}{F_{2}^{Z} F_{1}^{Z}} C_{m, n}\right]
$$


Stochastic volatility with heterogeneous time scales

$$
\begin{aligned}
& +e^{F_{1}^{Z} \tau}\left[-\frac{A_{2}^{Z}}{F_{2}^{Z}-F_{1}^{Z}}\left(C_{m, n+1}+\frac{A_{1}^{Z}}{F_{1}^{Z}} C_{m, n}\right)\right] \\
& +e^{F_{2}^{Z} \tau}\left[C_{m, n+2}+\frac{A_{2}^{Z}}{F_{2}^{Z}-F_{1}^{Z}}\left(C_{m, n+1}+\frac{A_{1}^{Z}}{F_{2}^{Z}} C_{m, n}\right)\right] .
\end{aligned}
$$

Computation of $f_{t}^{(m, n, 1,1)}(\tau)=\mathbb{E}\left[Y_{t}^{m} Z_{t}^{n} Y_{t+\tau} Z_{t+\tau}\right]$. The evolution of the joint process $Y_{t} Z_{t}$ is given by

$$
\begin{aligned}
\mathrm{d}\left(Y_{t} Z_{t}\right)= & \left(F_{1,1} Y_{t} Z_{t}+A_{1}^{Z} Y_{t}+A_{1}^{Y} Z_{t}\right) \mathrm{d} t \\
& +\sqrt{\sigma_{Y}^{2}} Y_{t} Z_{t} \mathrm{~d} W_{t}^{Y}+\sqrt{\sigma_{Z}^{2}} Y_{t} Z_{t} \mathrm{~d} W_{t}^{Z},
\end{aligned}
$$

and substitution inside the expectation gives

$$
\begin{aligned}
& f_{t}^{(m, n, 1,1)}(\tau)=\left[\frac{A_{1}^{Y} A_{1}^{Z}}{F_{1,1}}\left(\frac{1}{F_{1}^{Y}}+\frac{1}{F_{1}^{Z}}\right)\right] C_{m, n} \\
& -\frac{A_{1}^{Z}}{F_{1,1}-F_{1}^{Y}} e^{F_{1}^{Y} \tau}\left[C_{m+1, n}+\frac{A_{1}^{Y}}{F_{1}^{Y}} C_{m, n}\right] \\
& -\frac{A_{1}^{Y}}{F_{1,1}-F_{1}^{Z}} e^{F_{1}^{Z} \tau}\left[C_{m, n+1}+\frac{A_{1}^{Z}}{F_{1}^{Z}} C_{m, n}\right] \\
& +e^{F_{1,1} \tau}\left[C_{m+1, n+1}+\frac{A_{1}^{Z}}{F_{1,1}-F_{1}^{Y}} C_{m+1, n}+\frac{A_{1}^{Y}}{F_{1,1}-F_{1}^{Z}} C_{m, n+1}\right. \\
& \left.-A_{1}^{Y} A_{1}^{Z}\left(\frac{2 F_{1,1}-F_{1}^{Y}-F_{1}^{Z}}{F_{1,1}\left(F_{1,1}-F_{1}^{Y}\right)\left(F_{1,1}-F_{1}^{Z}\right)}\right) C_{m, n}\right] .
\end{aligned}
$$

As expected from the structure of model (4), and as confirmed by all previous examples, it is clear that the functions $f_{t}^{(m, n, p, q)}(\tau)$ admit a general expansion reading

$$
f_{t}^{(m, n, p, q)}(\tau)=\sum_{i=1}^{p} \sum_{j=1}^{q} h_{i, j}^{(m, n, p, q)}(t) e^{F_{i, j} \tau}
$$

where the terms $h_{i, j}^{(m, n, p, q)}(t)$ can be computed exactly. Coming back to Equation C.1 we have

$$
\begin{aligned}
& \frac{\mathbb{E}\left[\mathrm{d} X_{t}^{2} \mathrm{~d} X_{t+\tau}^{2}\right]}{\mathrm{d} t^{2}}= \\
& f_{t}^{(2,0,2,0)}(\tau)+f_{t}^{(0,2,2,0)}(\tau)+2 f_{t}^{(0,2,2,0)}(\tau)+f_{t}^{(2,0,0,2)}(\tau)+f_{t}^{(0,2,0,2)}(\tau) \\
& \left.+2 f_{t}^{(0,2,0,2)}(\tau)+2\left[f_{t}^{(2,0,1,1)}(\tau)+f_{t}^{(0,2,1,1)}(\tau)+2 f_{t}^{(0,2,1,1)}(\tau)\right] . \quad \text { (C. } 6\right)
\end{aligned}
$$

By means of Equations C.3 - C.5, and after defining the auxiliary variables

$$
\begin{array}{ll}
T_{1}=C_{2,0}+C_{0,2}+2 C_{1,1}, & T_{2}=C_{3,0}+C_{1,2}+2 C_{2,1}, \\
T_{2}^{*}=C_{0,3}+C_{2,1}+2 C_{1,2}, & T_{3}=C_{4,0}+C_{2,2}+2 C_{3,1}, \\
T_{3}^{*}=C_{0,4}+C_{2,2}+2 C_{1,3}, & T_{4}=C_{3,1}+C_{1,3}+2 C_{2,2},
\end{array}
$$

we can write the following final expression

$$
\begin{aligned}
\frac{\mathbb{E}\left[\mathrm{d} X_{t}^{2} \mathrm{~d} X_{t+\tau}^{2}\right]}{\mathrm{d} t^{2}}= & {\left[\frac{A_{2}^{Y} A_{1}^{Y}}{F_{2}^{Y} F_{1}^{Y}}+\frac{A_{2}^{Z} A_{1}^{Z}}{F_{2}^{Z} F_{1}^{Z}}+2 \frac{A_{1}^{Y} A_{1}^{Z}}{F_{1,1}}\left(\frac{1}{F_{1}^{Y}}+\frac{1}{F_{1}^{Z}}\right)\right] T_{1} } \\
& -e^{F_{1}^{Y} \tau}\left(T_{2}+\frac{A_{1}^{Y}}{F_{1}^{Y}} T_{1}\right)\left[\frac{A_{2}^{Y}}{F_{2}^{Y}-F_{1}^{Y}}+2 \frac{A_{1}^{Z}}{F_{1,1}-F_{1}^{Y}}\right]
\end{aligned}
$$


Stochastic volatility with heterogeneous time scales

$$
\begin{aligned}
& -e^{F_{1}^{Z} \tau}\left(T_{2}^{*}+\frac{A_{1}^{Z}}{F_{1}^{Z}} T_{1}\right)\left[\frac{A_{2}^{Z}}{F_{2}^{Z}-F_{1}^{Z}}+2 \frac{A_{1}^{Y}}{F_{1,1}-F_{1}^{Z}}\right] \\
& +e^{F_{2}^{Y} \tau}\left[T_{3}+\frac{A_{2}^{Y}}{F_{2}^{Y}-F_{1}^{Y}}\left(T_{2}+\frac{A_{1}^{Y}}{F_{2}^{Y}} T_{1}\right)\right] \\
& +e^{F_{2}^{Z} \tau}\left[T_{3}^{*}+\frac{A_{2}^{Z}}{F_{2}^{Z}-F_{1}^{Z}}\left(T_{2}^{*}+\frac{A_{1}^{Z}}{F_{2}^{Z}} T_{1}\right)\right] \\
& +2 e^{F_{1,1} \tau}\left[T_{4}+\frac{A_{1}^{Y}}{F_{1,1}-F_{1}^{Z}} T_{2}^{*}+\frac{A_{1}^{Z}}{F_{1,1}-F_{1}^{Y}} T_{2}\right. \\
& \left.+\frac{2 F_{1,1}-F_{1}^{Y}-F_{1}^{Z}}{F_{1,1}\left(F_{1,1}-F_{1}^{Y}\right)\left(F_{1,1}-F_{1}^{Z}\right)}\right] .
\end{aligned}
$$

Ultimately, evaluation of the volatility autocorrelation (6) requires to compute $\operatorname{Var}\left[\mathrm{d} X_{t}^{2}\right]=\mathbb{E}\left[\mathrm{d} X_{t}^{4}\right]-\mathbb{E}\left[\mathrm{d} X_{t}^{2}\right]^{2}$ which is given by

$$
3\left(C_{4,0}+4 C_{3,1}+6 C_{2,2}+4 C_{1,3}+C_{0,4}\right) \mathrm{d} t^{2}-\left(C_{2,0}+2 C_{1,1}+C_{0,2}\right)^{2} \mathrm{~d} t^{2} .
$$

\section{References}

[1] Mandelbrot B B 1963 J. Bus. 36 394-419

[2] Mandelbrot B B 1997 Fractals and Scaling in Finance (Springer-Verlag)

[3] Dacorogna M M, Müller U A, Nagler R J, Olsen R B and Pictet O V 1993 J. Int. Money Finance $12413-438$

[4] Ding Z, Granger C W J and Engle R F 1993 J. Empirical Finance 1 83-106

[5] Lux T and Marchesi M 1999 Nature 397 498-500

[6] Lux T and Marchesi M 2000 Int. J. Theoretical Appl. Finance 3 675-702

[7] Alfi V, Cristelli M, Pietronero L and Zaccaria A 2009 Eur. Phys. J. B 67 385-397

[8] Alfi V, Cristelli M, Pietronero L and Zaccaria A 2009 Eur. Phys. J. B 67 399-417

[9] Müller U A, Dacorogna M M, Davé R D, Olsen R B, Pictet O V and Ward J R 1994 Fractals and intrinsic time - a challenge to econometricians $B$. Lüthje vol 4 ed Zinsprognose E (Verband öffentlicher Banken, Bonn) pp 213-239

[10] Müller U A, Dacorogna M M, Davé R D, Olsen R B, Pictet O V and von Weizsäcker J E 1993 J. Empirical Finance 4 213-239

[11] Corsi F 2009 J. Financ. Economet. 7 174-196

[12] Lynch P E and Zumbach G O 2003 Quant. Finance 3 320-331

[13] Bouchaud J P 2001 Quant. Finance 1 105-112

[14] Miccichè S, Bonanno G, Lillo F and Mantegna R N 2002 Physica A 314 756-761

[15] Bates D 2000 Journal of Econometrics 94 181-238

[16] Delpini D and Bormetti G 2011 Phys. Rev. E 83041111

[17] Heston S L 1993 Rev. Financ. Stud. 6 327-343

[18] Stein E M and Stein J C 1991 Rev. Financ. Stud. 4 727-52

[19] Andersen T G, Davis R A, Kreiss J P and Mikosch (eds) T 2009 Handbook of Financial Time Series (Berlin: Springer-Verlag)

[20] Christoffersen P, Heston S and Jacobs K 2009 Management Science 55 1914-1932

[21] Corsi F and Renò R 2012 J. Bus. Econ. Stat. 3(3) 368-380

[22] Bouchaud J P and Potters M 2003 Theory of Financial Risk and Derivative Pricing: from Statistical Physics to Risk Management (Cambridge University Press)

[23] Wilke C, Altmeyer S and Martinetz T 1998 Large-scale evolution and extinction in a hierarchically structured environment Artificial Life VI, Proceedings of the Sixth International Conference on Artificial Life ed Adami C, Belew R K, Kitano H and E T C pp 266-272

[24] Bouchaud J P, Matacz A and Potters M 2001 Phys. Rev. Lett. 87228701

[25] Perelló J, Masoliver J and Bouchaud J P 2004 Appl. Math. Finance 11 27-50

[26] Guillaume D M, Dacorogna M M, Davé R D, Müller U A, Olsen R B and Pictet O V 1997 Finance and Stochastics 1 95-129

[27] Cont R, Potters M and Bouchaud J P 1997 Scaling in stock market data: stable laws and 
beyond Scale Invariance and Beyond ed Dubrulle B, Graner F and Sornette D (Les Ulis: EDP Sciences)

[28] Liu Y, Cizeau P, Meyer M, Peng C K and Stanley H E 1997 Physica A 245 437-440

[29] Muzy J F, Delour J and Bacry E 2000 Eur. Phys. J. B 17 537-548

[30] Cont R 2001 Quant. Finance $1223-236$

[31] Javaheri A 2005 Wilmott Magazine May/June 78-81

[32] Hansen L P 1982 Econometrica 50 1029-1054

[33] Hamilton J D 1994 Time Series Analysis (Princeton University Press)

[34] Zumbach G O 2011 Quant. Finance 11 101-113

[35] Zumbach G O 2012 Financ. Res. Lett. 9 144-156

[36] Mantegna R N and Stanley H E 2000 An Introduction to Econophysics: Correlations and Complexity in Finance (Cambridge: Cambridge University Press)

[37] Novikov E A 1965 Sov. Phys. JETP-USSR 20 1290-1294

[38] Perelló J and Masoliver J 2003 Phys. Rev. E 67037102 